helped by statements on the size of the population "at risk," and on whether the unit was providing primary, secondary, or tertiary care (or a combination of these). There was also some uncertainty about whether all cases were poststreptococcal: low serum complement activity was accepted as an alternative to a raised antistreptolysin titre in $14 \%$ of patients and other unknown provoking agents could have caused the glomerulonephritis in these cases. Despite these limitations this Australian survey is among the best studies reported.

Nevertheless, some uncertainties remain. Most patients are not Australians, and in some ethnic groups the natural history may be different. Prognosis may also differ with different streptococci: the late results of patients followed after an epidemic in Trinidad showed that only $1.4 \%$ of 722 patients had clinical evidence of chronic renal disease 7-12 years later, ${ }^{5}$ and in Venezuela ${ }^{6}$ the results were intermediate between the series so far discussed. The age of the patient may also be important: most of Baldwin's and all of the Australian patients were adult. In children the prognosis is more generally agreed to be benign if urinary abnormalities do not persist. ${ }^{78}$ The Australian series is, however, consistent with European experience-where only a few cases of chronic renal failure are caused by an earlier attack of clinically recognisable poststreptococcal glomerulonephritis and few cases of acute poststreptococcal glomerulonephritis proceed to renal failure. A bad prognosis can be suspected clinically by the severity of the presenting illness or by the continuation of clinical features. Nevertheless, some patients may continue with detectable clinical abnormalities and yet still have a good long-term prognosis. Possibly this could be detected by serial analysis of serum creatinine concentrations. ${ }^{9} \mathrm{~A}$ benign course would be associated with stable concentrations, while continuing loss of nephrons causes a progressive rise, initially within the normal range.

More patients will need to be followed over several decades before Baldwin's conclusions can be considered to apply only to his particular population. Total reassurance for those patients who have poststreptococcal glomerulonephritis and who have no persisting clinical features will not be possible until such studies establish that the benign prognosis, observed over ten years by Lien and colleagues, ${ }^{3}$ will persist over many decades.

No evidence in any of these series suggests that after the attack of acute glomerulonephritis antibiotic prophylaxis has anything to offer. Antibiotics probably do not influence prognosis in the acute phase of glomerulonephritis either, but penicillin should be given to eliminate the streptococcus. An adequate course is 10 days at a dose of $250 \mathrm{mg}$, six hourly, of penicillin $\mathrm{V}$ or an equivalent oral penicillin, or a "single shot" of benzathine penicillin $2 \cdot 4$ megaunits.

Poststreptococcal glomerulonephritis is not a common disease and, like other poststreptococcal syndromes, its incidence was probably decreasing even before antibiotics. When the diagnosis is made, however, there is an urgent need to allay (when possible) the fears of the patients and relatives that result from increased public awareness of the lethal nature of some kidney conditions.

1 Jacobs, C, et al, Proceedings of the European Dialysis and Transplant Association, 1977, 14, 4.

${ }^{2}$ Baldwin, D S, American fournal of Medicine, 1977, 62, 1.

${ }^{3}$ Lien, J W K, Mathew, T H, and Meadows, R, Quarterly fournal of Medicine, 1979, 189, 99.

4 Turner, A G, et al, British Medical fournal, 1977, 2, 29.

${ }^{5}$ Nissenson, A R, et al, American fournal of Medicine, 1979, 67, 255.

${ }^{6}$ Rodriguez-Hurbe, B, et al, Clinical Nephrology, 1976, 5, 197.

${ }^{7}$ Dodge, W F, et al, Medicine, Baltimore, 1968, 47, 227.

${ }^{8}$ Travis, L B, et al, Clinical Nephrology; 1973, 1, 169.

${ }^{9}$ Rutherford, W E, et al, Kidney International, 1977, 11, 62.

\section{Randomised controlled trials?}

Bernard Shaw is alleged to have attributed his vigour to controversy; Popper ${ }^{1}$ has said that theories justify our preference for them only if they can weather attempted refutations. Cranberg's defence of methods other than randomised controlled trials for resolving problems in medicine ( $\mathrm{p}$ 1265) should be considered in this spirit.

The problems most often studied by randomised controlled trials fall into two broad categories. Firstly, such trials are used to examine broad topics such as prevention of a second myocardial infarction and the treatment of mild hypertension. Many workers believe that because multiple, possibly interrelated, factors influence the outcome there is no alternative to the randomised controlled trial in these circumstancesyet it may be just this profusion of detail that weakens the method. As Black ${ }^{2}$ has argued, where there are many factors effective randomisation becomes impossibly complex and the design of a trial insufferably so. In such cases we may doubt whether the randomised trial ever settled a problem. Almost all reports of trials on these broad clinical issues were seen initially as apparently conclusive but later became the subject of debate-though they often highlighted difficulties and drew attention to factors previously underestimated.

The second type of problem for which randomised trials are usually required is assessing drug treatments. The phases through which a new drug passes have been described, conveniently briefly, by Dollery. ${ }^{3}$ Phase 1 poses the question "Has the new drug a pharmacological action that may be useful in treatment?" and necessarily requires a small open trial. If the results are hopeful, what then ? Publication at this point, as Cranberg says, citing a BMA panel, greatly increases the difficulty of setting up a controlled trial. The alternative to suppress publication and start a trial-is both selfish and counterproductive: the sooner a wide audience is made aware of a possible advance the sooner will the work be assessed; and, equally important, any spin-offs can be thought about and explored. Our current insistence on randomised controlled trials has undoubtedly had a salutary effect on loose thinking, but more than once this has been at the expense of progress.

In the second phase of assessment there is a shift in emphasis from "Does the drug work ?" to "How does it compare with other treatments in efficacy and safety?" At first sight a randomised controlled trial seems the obvious way to answer that question. But apart from the difficulty of ensuring homogeneity in the groups there are other problems. Whether or not we use a placebo for comparison is perhaps a sideissue; but many clinicians believe it to be unethical with certain classes of drug (though the Empire Rheumatism Council trial ${ }^{4}$ that finally sanctified gold as a treatment for rheumatoid arthritis is an example of a good trial, its use of placebo does seem questionable). Clinicians may be deterred by the laborious nature of a randomised controlled trial, which requires substantial back-up facilities not available everywhere. Furthermore, anyone who has conducted such a trial and has tried to explain the objectives to patients will know how it alters the relationship between doctor and patient. The knowledge that treatment is not to be determined by the doctor cannot enhance trust.

Even if we suppose that these difficulties can be circumvented the methods used in randomised controlled trials are often imprecise. As one example, measurement of 
change in the circumference of finger joints has become a standard method of assessment in rheumatology trials; yet it depends on selection of the right type of patient if it is to be effective.5 A different kind of misuse of randomised clinical trials is that they have become part of promotional campaigns; there is now a huge literature comparing antirheumatic drugs with each other. Yet papers can easily be selected to show that the effect of a drug $A$ is greater than drug $B$, which is greater than drug $C$, which is greater than drug A: such an Escher spiral must have required considerable effort by clinicians, but whose interests have been served?

So Cranberg's argument that retrospective controls may be valid is worth considering. Until recently this might have meant reliance on the memory of subjective evidence; but data are now collected in more objective form and, as Cranberg says, are more easily retrieved. To be sure, strictures about methods and their use in randomised controlled trials apply just as forcibly to the use of historical controls, but they do not sway the evidence in favour of the randomised trial. An argument often advanced against comparisons based on retrospective data is that patterns of disease change. But how rapid are such changes in relation to the periods concerned? And if change is rapid, perhaps for environmental reasons, then a randomised trial may be invalidated just as much as any other.

In his wide-ranging paper that includes comments on randomised controlled trials Black concludes that "in spite of these reservations, I would agree that if a controlled trial is practicable and can produce a result, it is a most valuable contribution to progress...." That and no more: the controlled trial has been placed on too high a pedestal and needs to be brought back to earth.

${ }^{1}$ Popper, K, Unended Quest: an Intellectual Autobiography. Fontana/ Collins, 1976.

2 Black, D, fournal of the Royal College of Physicians of London, 1979, $13,57$.

${ }^{3}$ Dollery, C T, fournal of the Royal College of Physicians of London, 1977, 11, 226.

${ }^{4}$ Empire Research Council, Annals of the Rheumatic Diseases, 1961, 20, 315.

${ }^{5}$ Hart, F D, and Huskisson, E C, Lancet, 1972, 1, 28.

\section{Who carries the can?}

Clinical medicine, and especially therapeutics, advances by a process of evolution, in which only the changes proved to be of value survive. New ideas and new drugs are introduced and assessed and either accepted into the body of orthodox medicine or rejected. In recent years, for example, betablockers and bypass grafting have become accepted treatments for coronary heart disease, while anticoagulants and hyperbaric oxygen have fallen into disfavour.

Unfortunately these evolutionary principles do not seem to be applied to changes in the organisation of medicine. Only rarely is any attempt made at a pilot study: more often a committee makes its report and new measures are introduced with no built-in system for their evaluation. An innovation may be introduced with all the fervour of a divine revelation - but the conviction of enthusiasts is a poor substitute for objective evidence based on scundly constructed experiments. More important, when practical experience suggests that an innovation does not work there is no mechanism for it to fade silently away.
So the disenchantment evident in Appleyard and Maden's criticism of multidisciplinary teams ( $p$ 1305) is not really surprising. These teams were introduced into clinical medicine as part of a new gospel-consensus management-which was envisaged as a bright new solution to some of the discontents in the Health Service. No doubt there are circumstances in which consensus management does work, but making clinical decisions is not one of them. As the authors explain, the fundamental defect in the concept of team management is that individuals will follow decisions with which they disagree only if they are forced to do so. If the team is arranged in the old-fashioned hierarchical fashion, with the doctor as boss, he can enforce such decisions; but in the current set-up a social worker or psychologist who disagrees with the team decision remains free to obstruct it by refusing, for example, to arrange for special education for a disturbed child. Neither the legal nor the ethical implications of obstruction of this kind have yet received enough attention.

Whatever the defects of the old system, in which the doctor was an autocrat who took advice from his colleagues but then made his own decision, it had two important merits. Firstly, the patient knew who was treating him. All too often nowadays patients are made confused and miserable by obvious conflicts and anomalies in their treatment by different health professionals. Secondly, when things went wrong, the doctor carried the can-and at worst had to appear in court and possibly pay damages or answer charges before the General Medical Council. Few of the new health professions have established procedures (comparable to those for doctors and nurses) for dealing with unprofessional or unethical conduct by their members. And where does the legal responsibility lie in these days of team management? The recent spate of civil liability cases in which damages have been awarded against health authorities suggests that they will be held liable for errors and omissions made by the teams they employ-but spreading responsibility so widely must encourage individuals to believe that they were not to blame. In traditional clinical medicine doctors (most often) learnt from their mistakes: the new-style medicine of modern management seems to be designed in such a way that no one has to admit that the mistake may have been his or her fault.

\section{Asymptomatic complete heart block}

The widespread practice of recording routine electrocardiograms in almost every patient attending the medical outpatient department has led to general physicians seeing many more asymptomatic patients with complete atrioventricular block. In these circumstances the risk of syncope in individual patients is unknown; but some clinicians have taken the view that it is always sufficient to warrant prophylactic insertion of a pacemaker.

Atrioventricular block may be due to lesions at any of three sites: the atrioventricular node, the bundle of $\mathrm{His}$, or the bundle branches. ${ }^{1}$ Structural abnormalities affecting the His-Purkinje system are generally believed to be progressive, so that Adams-Stokes attacks are more likely to develop in such cases; in contrast, abnormalities affecting the atrioventricular node have a more benign course. ${ }^{2}$ The introduction of His bundle electrography has allowed cardiologists to define 